\title{
The efficacy and duration of treatment with propranolol in children with cyclic vomiting syndrome in southern Iran
}

\author{
Mahmoud Haghighat ${ }^{1}$, Hajar Memari ${ }^{1}$, Naser Honar ${ }^{1}$, Seyed Mohsen Dehghani ${ }^{1}$, Mohammad-Hadi Imanieh ${ }^{1}$, \\ Seyed-Javad Injoo ${ }^{1}$, Hazhir Javaherizadeh ${ }^{2}$ \\ ${ }^{1}$ Gastroenterohepatology Research Center, Nemazee Teaching Hospital, Shiraz University of Medical Sciences, Shiraz, Iran \\ ${ }^{2}$ Research Center for Infectious Diseases of Digestive System [Alimentary Tract Research Center], Ahvaz Jundishapur University \\ of Medical Sciences, Ahvaz, Iran
}

Key words: cyclic vomiting syndrome, children, propranolol, duration of therapy, prevention.

Address for correspondence: Dr. Hazhir Javaherizadeh, Research Center for Infectious Diseases of Digestive System [Alimentary Tract Research Center], Ahvaz Jundishapur University of Medical Sciences, 6135873391 Ahvaz, Iran, phone: +98 9121231204, e-mail: hazhirja@ yahoo.com

\begin{abstract}
Introduction: Cyclic vomiting syndrome is a functional disorder characterised by repeated episodes of nausea and vomiting with symptom-free intervals between the attacks. Cyclic vomiting syndrome is a migraine equivalent; therefore, anti-migraine medications are effective for cyclic vomiting syndrome prophylaxis, but duration of treatment is not clear.

Aim: To determine the efficacy and duration of drug therapy in cyclic vomiting syndrome.

Material and methods: This study was conducted on 206 cases of cyclic vomiting syndrome, who were treated with propranolol as prophylaxis. After they were symptom-free for at least 9 months, propranolol was tapered and discontinued and then they were followed for at least 6 months after drug withdrawal for evaluation of recurrence.

Results: The study subjects included 127 boys and 79 girls. The mean age of symptom onset was 3.4 years (range: 3 months to 14.5 years) and the mean age at the time of diagnosis was 5.7 years (range: 8.4 months to 18 years). Among the 206 patients in whom propranolol was discontinued, only $16(7.8 \%)$ subjects developed recurrence of symptoms in the 6-month follow-up period.

Conclusions: There is no need to continue prophylaxis of cyclic vomiting syndrome for a long time. It is also possible to make the duration of drug therapy shorter, which is useful for the convenience of the patients and prevention of drug side effects.
\end{abstract}

\section{Introduction}

Cyclic vomiting syndrome (CVS) is a functional disorder for which multiple proposed mechanisms exist. The disorder is characterised by repeated episodes of nausea and vomiting accompanied by some other symptoms such as abdominal pain, headache, photophobia, diarrhoea, constipation, and fever. Each episode can last from a few hours to several days. Between the attacks, the patients are healthy and symptom-free [1-4]. Multiple triggering factors have been proposed for acute emetic episodes. Some of these factors are respiratory infections (upper respiratory tract infections and chronic sinusitis), emotional stress, motion sickness, insomnia, severe physical activity, certain food products (cacao, cheese), and menstruation in some women [5]. Cyclic vomiting syndrome was primarily described in children, but it may be diagnosed at any age [6-8]. The prevalence of CVS has been reported to be about 2\% [9-11]. There are some sets of criteria for diagnosis of CVS, one of which is the NASPGHAN (North American Society for Paediatric Gastroenterology, Hepatology, and Nutrition) consensus statement, presented in 2008 as new clinical criteria for CVS, and which can be used for both children and adults [12]. Episodes of vomiting in CVS are often self-limiting and improve without treatment; however, the duration of the attacks is unpredictable and if prolonged, it can cause dehydration, electrolyte imbalance, and related complications [13]. Therefore, CVS should be prevented if episodes occur frequently and if they negatively impact the quality of life. Nevertheless, types of effective treatment and duration of therapy are very controversial [14]. Cyclic vomiting syndrome is thought 
to be a migraine equivalent $[15,16]$ and will frequently progress to migraine headaches later in life. A family history of migraine headaches is often present [17]. Thus, anti-migraine medications are considered to be effective for CVS prophylaxis [18]. One such medication is propranolol, which is frequently used for prevention of CVS attacks [19].

However, to the best of our knowledge, the duration of CVS prophylaxis has not been mentioned in the literature and most physicians continue medication until adolescence. Although propranolol is a safe drug in children, every medication can cause side effects in some individuals.

\section{Aim}

The aim of the present study was to evaluate the duration of prophylactic drug therapy for CVS.

\section{Material and methods}

The present study was conducted on 242 new cases of CVS, who met NASPGHAN criteria for CVS and were followed by paediatric gastroenterologists (authors).

NASPGHAN consensus criteria for CVS include the presence of all of the following:

1. At least five attacks in any interval, or a minimum of three attacks during a 6-month period.

2. Episodic attacks of intense nausea and vomiting lasting from $1 \mathrm{~h}$ to 10 days and occurring at least 1 week apart.

3. Stereotypical pattern and symptoms in the individual patient.

4. Vomiting during attacks occurs at least four times per hour for at least $1 \mathrm{~h}$.

5. Return to baseline health between episodes.

6. Not attributed to another disorder [12].

For each subject, a standardised questionnaire including demographic data, associated conditions, clin-

Table I. Features of the cases

\begin{tabular}{l} 
Sex: male $61.7 \%$, female $38.3 \%$ \\
\hline Mean age at onset: $3.4 \pm 3.02$ years ( 3 months -14.5 years) \\
\hline Mean age at diagnosis: $5.7 \pm 3.2$ years ( 8.4 months -18 years) \\
\hline Associated symptoms: \\
Abdominal pain: $142(68.9 \%)$ \\
\hline Diarrhoea: $32(15.5 \%)$ \\
Fever: $22(10.7 \%)$ \\
Headache: 17 (8.3\%) \\
Constipation: $4(1.9 \%)$
\end{tabular}

ical features, and management was completed. After diagnosis of CVS, propranolol with $1 \mathrm{mg} / \mathrm{kg}$ of body weight per day was started for all of the patients.

The individuals were regularly followed and if the response was not favourable, the drug dose was increased to 2 and finally $3 \mathrm{mg} / \mathrm{kg} /$ day. Patients who suffered from asthma, diabetes mellitus, cardiovascular diseases such as sinus bradycardia or decompensated heart failure, and drug hypersensitivity were excluded from the study. A sinus rate less than 90 beats/min in neonates and less than 60 beats/min thereafter is considered to be sinus bradycardia. Those who developed propranolol side effects, such as hypotension, bradycardia, hypoglycaemia, bronchospasm, or wheezing attacks were excluded as well.

Another exclusion criterion was lack of response to propranolol during a 3-month period.

In this study, response to medication was defined as at least $50 \%$ reduction in the severity and frequency of the attacks. In patients who were symptom-free for at least 9 months, propranolol was tapered by $25 \%$ dose reduction every 20 days, and finally discontinued. After that, they had regular visits at 1, 3, and 6 months after discontinuation of the treatment. In each visit, they were evaluated for recurrence of the symptoms. During the follow-up period, patients who again experienced a CVS attack severe enough to restart treatment were considered as cases of drug withdrawal failure.

\section{Ethics}

This study was approved by the Institutional Review Board of Shiraz University of Medical Sciences, SUMS, Iran, and written informed consent was obtained from all the study participants.

\section{Statistical analysis}

Statistical variables were described by means and standard deviations for continuous variables, and by frequencies and percentages for categorical variables. The data were analysed with Statistical Package for the Social Sciences for Windows, version 15 (SPSS Inc., Chicago, Ill., USA) using $t$-test, $\chi^{2}$ test, and descriptive statistical methods. $P$-values less than 0.05 were considered statistically significant.

\section{Results}

Demographic data of 206 patients were analysed. The majority of the patients were male (61.7\%). The mean age at the onset of symptoms was 3.4 years with standard deviation of 3.02 (range: 3 months to 14.5 years), and the mean age at the time of diagnosis of CVS was $5.7 \pm 3.2$ years (range: 8.4 months to 18 years). 
All cases had vomiting during their attacks. The most common associated symptom was abdominal pain, which was positive in $142(68.9 \%)$ patients. Other accompanying symptoms were diarrhoea, fever, headache, and constipation in 32 (15.5\%), 22 (10.7\%), 17 (8.3\%), and 4 (1.9\%) participants, respectively (Table I, Figure 1).

The mean duration of the follow-up after discontinuation of propranolol was 9 months (range: 6-12 months).

Twenty-one patients were excluded due to lack of follow-up, drug side effect, or changing the diagnosis. Among the study cases, one developed wheezing attacks and bronchospasm due to propranolol; consequently, the drug was discontinued and the patient was excluded from the study. Also, due to the lack of response to treatment in another patient, additional evaluation was done and the diagnosis was changed to ureteropelvic junction obstruction (UPJO), so the patient was excluded from the study, and 19 cases did not refer for follow-up and were not available by telephone.

Furthermore, due to lack of appropriate response to propranolol, we could not discontinue the medication in 15 patients until the end of the study

Therefore, they were all excluded from the study. Finally, we were able to discontinue propranolol with proper follow-up for at least 6 months in 206 patients.

Among 221 patients who received propranolol and had regular follow-up, 206 (93.2\%) subjects responded ideally to the medication. From 206 patients in whom propranolol was discontinued, only 16 (7.8\%) had recurrence of the symptoms during the follow-up period. The study results revealed no significant difference between the patients in whom drug discontinuation was successful and those with recurrence of the symptoms after drug withdrawal regarding sex distribution $(p=0.942)$, mean age $(p=0.6)$, duration of attacks $(p=0.408)$, interval between episodes $(p=0.699)$, history of motion sickness ( $p=0.750)$, and family history of migraine headache $(p=0.781)$.
$P$-values less than 0.05 were considered statistically significant.

\section{Discussion}

Cyclic vomiting syndrome is a chronic, non-organic disorder with unknown cause. This disorder is characterised by sudden, recurrent, and similar episodes of vomiting.

In our study, all of the children had nausea and vomiting that was similar to other studies. Nausea is the most distressing symptom in children with CVS [20]: It is recurrent and paroxysmal, is not completely stopped by vomiting, and disappears only with sleeping or when the episode is over. It seems that some behavioural symptoms in children with CVS (e.g. foetal positioning, social withdrawal), turning off lights and televisions may be effective in control of severe nausea [21].

In our study the most common concomitant symptom was abdominal pain, which was detected in $69 \%$ (142 cases) and then diarrhoea, fever, and headache. This is in agreement with other studies. In addition to vomiting, other Gl symptoms may present in patients with CVS.

Eighty percent of children have abdominal pain that can be severe enough and mimic surgical abdomen and finally lead to laparotomy. Patients may also have epigastric pain secondary to peptic injury of the oesophagus. Also, substernal pain secondary to esophagitis may occur.

$\mathrm{Li}$ and Misiewicz reported abdominal pain in $81 \%$ of their patients [20]. This association can result in many unnecessary diagnostic tests and even surgical intervention before the diagnosis of CVS is established.

In a study of 41 cases of CVS, authors reported 17 surgical attempts on 16 patients before CVS diagnosis [20].

Fever, diarrhoea, or both are noted in approximately one third of CVS patients, complicating the differentiation of this condition from gastroenteritis. These common findings are probably due to associated autonomic

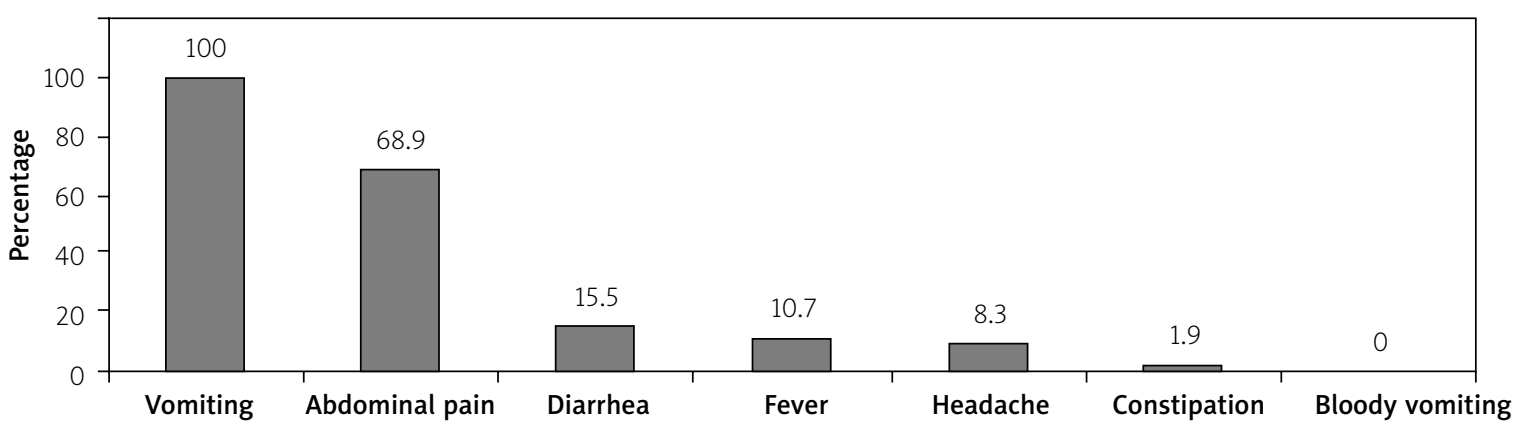

Figure 1. Frequency of concomitant symptoms in children with CVS 
symptoms, especially pallor and lethargy [20]. Lethargy can be very severe, and finally they may be unable to talk or walk or may appear comatose.

Another symptom is salivation (27\%), which may be significant [20].

The pathophysiology is unknown, but a genetic component may be involved, with evidence of mitochondrial heteroplasmies that predispose to CVS and other related disorders (e.g. migraine and chronic fatigue syndrome).

Other theories about what may be involved in the pathogenesis of CVS include autonomic dysfunction and, possibly, corticotropin-releasing factor (CRF) because stress is a risk factor for triggering an attack. The possible role of cannabis in causing CVS has been examined, but it remains controversial.

According to guidelines formulated by NASPGHAN, cyproheptadine is recommended as the first-line choice in children younger than 5 years.

However, cyproheptadine can cause an increase in appetite and eventually lead to weight gain. In children more than 5 years old and adolescents, amitriptyline is recommended as the first-line therapy. Propranolol has been proposed as the second choice for CVS prophylaxis $[12,14]$.

In these studies, the response rate of tricyclic antidepressants (TCAs) are about 80\% [8]. However, in our previous study, which was done on 181 cases of CVS, $92 \%$ of patients who received propranolol had a good response without any significant side effects, indicating that propranolol is very effective and safe for CVS prophylaxis [22].

Also, in the present study, propranolol was administered as the first-line prophylaxis, and $93.2 \%$ of patients responded to it; this is a significant result as compared other studies. In the NASPGHAN consensus statement, it was mentioned that propranolol has moderate efficacy in paediatric CVS (35-75\% in four studies) [12]. Sunku puts forward the figures of $52-65 \%$ [2], and Li and Misiewicz 50\% [20]. All these figures are well below the percentage in our study $(93.2 \%)$.

In an open-label study of 41 patients with CVS, who were followed-up for 1-2 years, long-term therapy with TCAs significantly reduced the frequency and duration of episodes and the number of hospitalisations [8].

In this study, $80 \%$ of patients reported overall improvement of symptoms; however, one third of the patients reported mild adverse effects that did not lead to discontinuance of the medication.

Also, other studies showed that TCAs are effective in CVS with a response rate of about $80 \%$ [23, 24]. In one study by Lee et al. TCAs had good efficacy in reducing the frequency and duration or the intensity of attacks [19].
However, TCAs and cyproheptadine have multiple side effects in children, and the patients may not take these medicines as well.

The side effects of cyproheptadine include central nervous system (CNS) depression, drowsiness, sedation ranging from mild drowsiness to deep sleep (most frequent), dizziness, tremor, delirium, seizures, and toxic psychosis.

Also, this drug can cause other complications including muscular, cardiovascular, GI, hepatic, renal, and respiratory side effects.

Additionally, TCAs have several side effects among cardiac arrhythmias, especially with overdose, and monitoring of the QT interval before therapy and in follow-up is advised. Other side effects of TCAs are neurological complications (including hallucination, delirium, coma), constipation, and urinary retention [8].

Thus, according to our studies, which were conducted in this centre and concluded in previous paper [22], the response rate of propranolol was significantly greater than other reports and can be used as the first-line choice in children. Therefore, we recommend propranolol as the drug of choice for this syndrome in children.

The reason for the significant difference in response to propranolol in our centre compared to other centres is not clear and may be due to differences in response to treatment in different regions, or the dose of propranolol, which may be higher in our centre.

Considering the negative results of upper gastrointestinal series with small bowel follow through (UGISBFT) in $97 \%$ of the cases in our previous study [22], no additional workup was done for the patients who fulfilled the clinical criteria of CVS and had no alarm sign or symptom in the history and physical examination in the present study.

However, NASPGHAN consensus recommends UGI-SBFT followed by empiric anti-migraine treatment without extensive diagnostic evaluation [12].

In our previous report, the medication was discontinued in 52 (31\%) patients who were asymptomatic with propranolol for at least 5 years. These children had follow-up for 1 year with recurrence in only 2 (3.8\%) cases [22]. In the present study, we shortened the duration of treatment to 9 months after being asymptomatic and noticed that only $7.8 \%$ of the subjects developed recurrence of attacks. There is no statistically significant difference between these two groups regarding drug withdrawal failure $(p=0.48)$.

The results suggest that it may be possible to shorten the duration of CVS prophylaxis. This may be beneficial for patients and families in order to prevent drug side effects. In this study, no significant difference was found between the subjects with successful drug with- 
drawal and those in whom treatment discontinuation failed regarding sex, age, disease severity, history of motion sickness, and family history of migraine. The reason why some patients remained asymptomatic and some experienced episodes of vomiting after holding propranolol were not clear for us and merits further studies.

Limitation: single-centre study.

\section{Acknowledgments}

This article was done in Shiraz University of Medical Sciences. The authors would like to thank the Research Affairs of Shiraz University of Medical Sciences for support with the grant number: 90-01-01-2735. We would also like to thank Dr. Nasrin Shokrpour and Ms. A. Keivanshekouh for editorial assistance and Ms. Rosta for statistical analysis at the Centre for Development of Clinical Research of Nemazee Hospital.

\section{Conflict of interest}

The authors declare no conflict of interest.

\section{References}

1. Lee LY, Abbott L, Moodie S, et al. Cyclic vomiting syndrome in 28 patients: demographics, features and outcomes. Eur J Gastroenterol Hepatol 2012; 24: 939-43.

2. Sunku B. Cyclic vomiting syndrome: a disorder of all ages. Gastroenterol Hepatol 2009; 5: 507-15.

3. Lin YP, Ni YH, Weng WC, et al. Cyclic vomiting syndrome and migraine in children. J Formos Med Assoc 2011; 110: 382-7.

4. Hejazi RA, McCallum RW. Cyclic vomiting syndrome in adults: rediscovering and redefining an old entity. Aliment Pharmacol Ther 2011; 34: 263-73.

5. Shin YK, Kwon JG, Kim KY, et al. A case of cyclic vomiting syndrome responding to gonadotropin-releasing hormone analogue. J Neurogastroenterol Motil 2010; 16: 77-82.

6. Pareek N, Fleisher DR, Abell T. Cyclic vomiting syndrome: what a gastroenterologist needs to know. Am J Gastroenterol 2007; 102: $2832-40$

7. Erturk O, Uluduz D, Karaali-Savrun F. Efficacy of nebivolol and amitriptyline in the prophylaxis of cyclic vomiting syndrome: a case report. Neurologist 2010; 16: 313-4.

8. Hejazi RA, Reddymasu SC, Namin F, et al. Efficacy of tricyclic antidepressant therapy in adults with cyclic vomiting syndrome: a two-year follow-up study. I Clin Gastroenterol 2010; 44: 18-21.

9. Forbes D, Fairbrother S. Cyclic nausea and vomiting in childhood. Aust Fam Physician 2008; 37: 33-6.

10. Olmez A, Kose G, Turanli G. Cyclic vomiting with generalized epileptiform discharges responsive to topiramate therapy. Pediatr Neurol 2006; 35: 348-51.

11. Yang HR. Recent concepts on cyclic vomiting syndrome in children. J Neurogastroenterol Motil 2010; 16: 139-47.

12. Li BU, Lefevre F, Chelimsky GG, et al. North American Society for Pediatric Gastroenterology, Hepatology, and Nutrition consensus statement on the diagnosis and management of cyclic vomiting syndrome. J Pediatr Gastroenterol Nutr 2008; 47: 379-93.

13. Chow S, Goldman RD. Treating children's cyclic vomiting. Can Fam Physician 2007; 53: 417-9.

14. Sudel B, Li BU. Treatment options for cyclic vomiting syndrome. Curr Treat Options Gastroenterol 2005; 8: 387-95.

15. Paul SP, Barnard P, Soondrum K, et al. Antimigraine (lowamine) diet may be helpful in children with cyclic vomiting syndrome. J Pediatr Gastroenterol Nutr 2012; 54: 698-9.

16. Abraham MB, Porter P. Clonidine in cyclic vomiting. J Pediatr Gastroenterol Nutr 2011; 53: 219-21.

17. Haan J, Kors EE, Ferrari MD. Familial cyclic vomiting syndrome. Cephalalgia 2002; 22: 552-54.

18. Hikita T, Kodama H, Kaneko S, et al. Sumatriptan as a treatment for cyclic vomiting syndrome: a clinical trial. Cephalalgia 2011; 31: 504-7.

19. Lee LY, Abbott L, Mahlangu B, et al. The management of cyclic vomiting syndrome: a systematic review. Eur I Gastroenterol Hepatol 2012; 24: 1001-6.

20. Li BU, Misiewicz L. Cyclic vomiting syndrome: a brain-gut disorder. Gastroenterol Clin North Am 2003; 32: 997-1019.

21. Li BU, Balint JP. Cyclic vomiting syndrome: evolution in our understanding of a brain-gut disorder. Adv Pediatr 2000; 47: 117-60.

22. Haghighat $M$, Rafie $S M$, Dehghani $S M$, et al. Cyclic vomiting syndrome in children: experience with 181 cases from southern Iran. World J Gastroenterol 2007; 13: 1833-6.

23. Boles RG, Lovett-Barr MR, Preston A, et al. Treatment of cyclic vomiting syndrome with co-enzyme Q10 and amitriptyline, a retrospective study. BMC Neurol 2010; 10: 10.

24. Hejazi RA, Lavenbarg TH, Foran P, et al. Who are the nonresponders to standard treatment with tricyclic antidepressant agents for cyclic vomiting syndrome in adults? Aliment Pharmacol Ther 2010; 31: 295-301.

Received: 5.09.2016

Accepted: 18.11.2016 